\title{
IncRNA LINC00673 induces proliferation, metastasis and epithelial-mesenchymal transition in thyroid carcinoma via Kruppel-like factor 2
}

\author{
ERJIE XIA* , ADHEESH BHANDARI*, YANYAN SHEN, XIAOFEN ZHOU and OUCHEN WANG \\ Department of Thyroid and Breast Surgery, The First Affiliated Hospital of Wenzhou Medical University, \\ Wenzhou, Zhejiang 325000, P.R. China
}

Received May 14, 2018; Accepted August 9, 2018

DOI: $10.3892 /$ ijo.2018.4524

\begin{abstract}
The incidence of thyroid cancer has increased in the past decades; however, the underlying molecular mechanisms of thyroid cancer tumorigenesis remain unknown. Using sequencing technology, long intergenic non-protein coding RNA 673 (LINC00673) was identified to be upregulated in several tumor tissues. However, the biological role of LINC00673 in thyroid carcinoma has yet to be determined. In this study, 60 matched pairs of thyroid tumor tissue and normal tissue were selected for study using reverse transcription-quantitative polymerase chain reaction (RT-qPCR) to validate previous findings; then, clinicopathologic features of the tissues were analyzed. Proliferation, colony formation, migration and invasion assays were performed, and epithelial-mesenchymal transition (EMT)associated phenotypes were investigated following transfection with small interfering RNA to determine the specific role of LINC00673 in thyroid carcinoma cell lines (TPC1, KTC-1 and BCPAP). The study revealed that long non-coding RNA LINC00673 was significantly upregulated in thyroid cancer tissues compared with paired adjacent non-tumor tissues using RT-qPCR and that high expression of LINC00673 is was associated with larger tumor size and lymph node metastasis in the validated cohort. Knockdown of LINC00673 inhibited cell proliferation and metastasis, whereas, LINC00673 overexpression had the opposite effect. The results showed that LINC00673 may influence EMT and the expression of Kruppel-like factor 2 (KLF2). Notably, KLF2 is considered a tumor suppressor gene in a variety of tumors. Finally, knock down of KLF2 enhanced thyroid carcinoma cell proliferation, and invasion and migration. In this study, the function of LINC00673 in promoting the
\end{abstract}

Correspondence to: Professor Ouchen Wang, Department of Thyroid and Breast Surgery, The First Affiliated Hospital of Wenzhou Medical University, Wenzhou, Zhejiang 325000, P.R. China

E-mail: woc863@hotmail.com

*Contributed equally

Key words: papillary thyroid carcinoma, long intergenic non-protein coding RNA 673, proliferation, metastasis, Kruppel-like factor 2 proliferation and metastasis of thyroid carcinoma cell lines was identified, and LINC00673 may act as a novel therapeutic target for treating thyroid carcinoma.

\section{Introduction}

Thyroid cancer is one of the most common malignancies in the world with an estimated 53,990 new cases and 2,060 mortalities expected in the USA in 2018 (1). Thyroid carcinoma is considered a well-differentiated type of thyroid cancer and accounts for $\sim 80 \%$ of all types of thyroid cancer (2). Although patients with thyroid carcinoma tend to have a good prognosis, a number of patients die from cancer metastasis and proliferation in a short time (3). With the development of medical and technical tools, numerous studies on thyroid carcinoma have been published in the past decades (4-6). However, the underlying mechanisms of thyroid carcinoma remain unclear.

With the progress made in whole genome sequencing technologies, long non-coding RNAs (lncRNAs) have attracted increasing attention from the scientific community (7-9). IncRNAs are a group of RNAs $>200$ nucleotides in length that have lost the ability to encode proteins (10). Accumulating evidence indicates that dysregulation of IncRNAs has a major role in the physiological progression of carcinogenesis. Gou et al (11) reported that high expression of IncRNA PVT1 promotes hepatocellular carcinoma progression by influencing microRNA (miR)-214. lncRNA SPRY4 intronic transcript 1 (SPRY4-IT1) has a lower expression level in lung adenocarcinomas than in normal tissue, and dysregulation of lncRNA SPRY4-IT1 alters the invasion and migration abilities of cancer cells (12). In addition, a series of reports have indicated that lncRNAs have crucial roles in the pathogenesis of thyroid cancer (13-15). However, the association between thyroid carcinoma and IncRNAs requires further study.

As a newly discovered IncRNA encoded by chromosome 17q25.1, long intergenic non-protein coding RNA 673 (LINC00673) lacks the ability to encode a protein. Lu et al (16) reported that LINC00673 is upregulated in non-small cell lung cancer, and that it sponges miR-150-5p and modulates ZEB1 indirectly. Additionally, LINC00673 may be a prognostic indicator of overall survival in breast cancer (17). Yu et al (18) demonstrated that overexpression of LINC00673 is associated with poor prognosis, and promotes invasion and metastasis in 
tongue squamous cell carcinoma. Unfortunately, there are no studies that describe the role of LINC00673 in thyroid cancer.

In a previous unpublished study, whole-transcriptome resequencing of 19 paired thyroid carcinoma tissue samples was performed and revealed that LINC00673 was upregulated in the tumor tissues compared with non-tumor tissues. In this study, 58 matched pairs of thyroid carcinoma and adjacent normal tissues were examined to further validate the dysregulation of LINC00673. The function of LINC00673 in thyroid carcinoma cell lines was also investigated. This study aimed to determine the role of LINC00673 in thyroid carcinoma.

\section{Materials and methods}

Patients and thyroid tissue samples. This study examined 60 matched pairs of thyroid carcinoma and adjacent non-tumor tissue samples (age range, 27-81 years; male/female, 3:2) resected in the Department of Thyroid and Breast Surgery at The First Affiliated Hospital of Wenzhou Medical University (Wenzhou, China) between February 2016 and July 2017. Fresh thyroid carcinoma tissues and non-tumor tissues were snap-frozen in liquid nitrogen. All patient-derived information was recorded following the protocols approved by the ethical standards of the Ethics Committee of the First Affiliated Hospital of Wenzhou Medical University. Written informed consent was obtained from each individual participant. The Kruppel-like factor 2 (KLF2) reads per kilobases per million reads expression value was obtained from The Cancer Genome Atlas (TCGA) portal. Sequence data from 502 thyroid carcinomas complete clinical features and 57 normal thyroid tissues was selected.

Cell culture and growth conditions. TPC1, KTC-1, BCPAP and HTORI-3 were received from Professor Mingzhao Xing of Johns Hopkins University School of Medicine (Baltimore, MA, USA). The TPC1, KTC-1 and BCPAP cell lines were cultured in RPMI-1640 (Invitrogen; Thermo Fisher Scientific, Inc., Waltham, MA, USA) containing $10 \%$ fetal bovine serum (FBS; Invitrogen; Thermo Fisher Scientific, Inc.), $100 \mathrm{mg} / \mathrm{ml}$ streptomycin and double-antibiotic $100 \mathrm{U} / \mathrm{ml}$ penicillin (Invitrogen; Thermo Fisher Scientific, Inc.). All thyroid cancer cell lines were incubated under an atmosphere of air containing $5 \% \mathrm{CO}_{2}$ at $37^{\circ} \mathrm{C}$.

Cell transfection. Thyroid cell line transfection was conducted using small interfering RNA (siRNA) and Lipofectamine ${ }^{\circledR}$ RNAiMAX transfection reagent (Invitrogen; Thermo Fisher Scientific, Inc.) following the manufacturer's instructions. Cells were plated in a 6 -well plate $24 \mathrm{~h}$ before transfection. TPC1 $\left(8 \times 10^{4}\right)$, BCPAP or KTC-1 $\left(2 \times 10^{5}\right)$ cells were transfected using $10 \mu \mathrm{l}$ (TPC1), $7.5 \mu \mathrm{l}$ (KTC-1) or $5 \mu \mathrm{l}$ (BCPAP) siRNA $(20 \mu \mathrm{M})$ and $4 \mu \mathrm{l}$ RNAiMAX (Thermo Fisher Scientific, Inc.) in a 6-well plate according to the manufacturer's recommendations. The siRNA sequences used in the study were as follows: LINC00673 siRNA-1, forward 5'-GCCUUUGUACUCAG CAAUUTT-3' and reverse 5'-AAUUGCUGAGUACAAAGG CTT-3'; LINC00673 siRNA-2, forward 5'-GGAUACAGAGU GAAUAGUUTT-3' and reverse 5'-AACUAUUCACUCUGUA UCCTT-3'; KLF-2 siRNA, forward 5'-GGGCCUUAAUUUG UACUGUTT-3' and reverse 5'-ACAGUACAAAUUAAGGCC CTT-3'. si-NC, forward 5'-UUCUCCGAACGUGUCACG UTT-3' reverse 5'-ACGUGACACGUUCGGAGAATT-3'. All
siRNAs were provided by Shanghai GenePharma Co., Ltd. (Shanghai, China).

$R N A$ extraction and reverse transcription-quantitative polymerase chain reaction ( $R T-q P C R)$. The total RNA from tissues and cells was isolated using TRIzol reagent (Invitrogen; Thermo Fisher Scientific, Inc.). The total RNA samples were reverse transcribed by the ReverTra Ace qPCR RT Kit (Toyobo Life Science, Osaka, Japan) according to the manufacturer's protocol. qPCR reactions were performed using a real-time PCR system (Applied Biosystems 7500; Applied Biosystems; Thermo Fisher Scientific, Inc.) using the Thunderbird SYBR qPCR Mix (Toyobo Life Science). The primer sequences used were as follows: LINC00673, forward 5'-TGCTGATGACACA TACACA-3' and reverse 5'-GACAAGGATGAACCATGA TAG-3'; KLF-2, forward 5'-CTGCACATGAAACGGCA CAT-3' and reverse 5'-CAGTCACAGTTTGGGAGGGG-3'; GADPH, forward 5'-GTCTCCTCTGACTTCAACAGCG-3' and reverse 5'-ACCACCCTGTTGCTGTAGCCAA-3'.

The relative expression of genes was normalized to GADPH expression and calculated according to the $2^{-\Delta \Delta \mathrm{Cq}}$ method described by Livak and Schmittgen (19).

Invasion and migration assays. In Transwell assays, KTC-1 $\left(5 \times 10^{4}\right.$ cells $)$ and BCPAP $\left(5 \times 10^{4}\right.$ cells $)$ were seeded in the upper chamber with $10 \%$ fetal bovine serum and $600 \mu \mathrm{l}$ medium containing $20 \%$ FBS was added to the lower chamber. After 20-24 h, the membranes were fixed with $4 \%$ paraformaldehyde for $30 \mathrm{~min}$ and stained with $0.4 \%$ crystal violet solution for $30 \mathrm{~min}$ at $37^{\circ} \mathrm{C}$. Cell migration and invasion abilities were evaluated by counting the cells. Five random fields of view were selected to count the cell numbers, and images were captured under a light microscope at a magnification of x 20 .

Cell proliferation assays. In the colony formation assay, TPC1 cells $\left(2 \times 10^{3}\right.$ cells $)$, KTC- 1 cells $\left(3 \times 10^{3}\right.$ cells $)$ and BCPAP cells $\left(3 \times 10^{3}\right.$ cells) were plated in 6 -well plates. The cells were maintained in RPMI-1640 with 10\% FBS for 7 days and then fixed with $4 \%$ paraformaldehyde for $30 \mathrm{~min}$ and stained with $0.4 \%$ crystal violet solution for $30 \mathrm{~min}$ at $37^{\circ} \mathrm{C}$. Images were captured using a camera.

In the CCK- 8 assay, TPC 1 cells $\left(2 \times 10^{3}\right.$ cells), KTC- 1 cells $\left(3 \times 10^{3}\right.$ cells $)$ and BCPAP cells $\left(3 \times 10^{3}\right.$ cells $)$ were plated in 96-well plates for $24 \mathrm{~h}$ and then transfected with siRNA. The cells were incubated for $24,48,72$ or $96 \mathrm{~h}$. All experiments were performed in triplicate.

Western blot analysis. Protein extraction buffers (radioimmunoprecipitation assay and phenylmethane sulfonyl fluoride; Sigma-Aldrich; Merck KGaA, Darmstadt, Germany) were added to the cells, and the protein concentrations were quantified using a bicinchoninic acid (BCA) kit. The samples were then homogenized, centrifuged at $12,000 \mathrm{x} \mathrm{g}$ at $4^{\circ} \mathrm{C}$ for $30 \mathrm{~min}$, then subjected to ultra-sonication (each action $5 \mathrm{sec}$, intermittent $6 \mathrm{sec}$ and each specimen 5 times). The supernatant was removed via centrifugation at $4,000 \times \mathrm{g}$ at $4{ }^{\circ} \mathrm{C}$ for $20 \mathrm{~min}$. The sample was used to perform quantification of the protein expression with BCA. According to the manufacturer's protocol, $20 \mu \mathrm{g}$ from each sample were separated by $10 \%$ SDS-polyacrylamide gel electrophoresis (Bio-Rad 
A

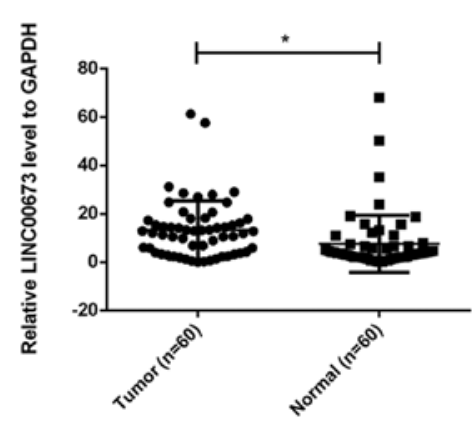

C

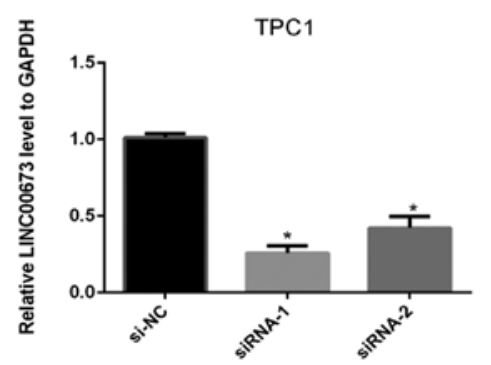

B

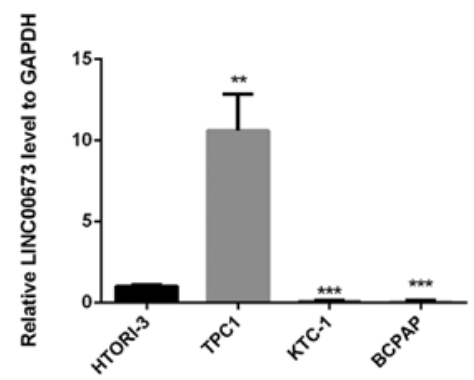

D

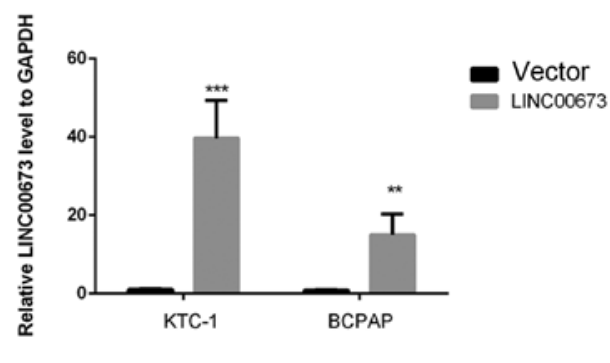

Figure 1. Expression of LINC00673 in a thyroid carcinoma cohort and in thyroid carcinoma cell lines. (A) LINC00673 expression was detected by RT-qPCR in 58 matched pairs of thyroid carcinoma and adjacent non-tumor tissues (paired t-test, ${ }^{*} \mathrm{P}<0.05$ ). (B) Relative expression of LINC00673 (normalized to the GAPDH gene) was examined via RT-qPCR. Compared with normal thyroid cell lines, the TPC1 cell line had higher LINC00673 expression, while the KTC-1 and BCPAP cell lines had lower expression. (C) The relative expression of LINC00673 (compared with the GAPDH gene) in the TPC1 cell line. Compared to the control group, the expression of LINC00673 was lower in the SiRNA group. (D) The relative expression of LINC00673 (compared with the GAPDH gene) in KTC-1 and BCPAP cell lines. Compared with the control group, the expression of LINC00673 in the LINC00673 group was higher. ${ }^{*} \mathrm{P}<0.05{ }^{* * *} \mathrm{P}<0.01,{ }^{* * * *} \mathrm{P}<0.001$ vs. treated with si-NC or a vector control using one-way analysis of variance. RT-qPCR, reverse transcription-quantitative polymerase chain reaction.

Laboratories, Inc., Hercules, CA, USA) and then transferred to PVDF membranes (EMD Millipore, Billerica, MA, USA). PVDF membranes were then incubated with blocking buffer (PBS with 5\% non-fat milk and $0.1 \%$ Tween-20) for $1 \mathrm{~h}$ at room temperature. Membranes were subsequently washed three times with Tris-buffered saline/Tween-20 for 5 min each and incubated overnight with primary antibodies at $4^{\circ} \mathrm{C}$.

The primary antibodies used in this study were as follows: N-cadherin (cat no. 13116; 1:1,000 dilution; Cell Signaling Technology, Inc., Danvers, MA, USA), vimentin (cat. no. 5741; 1:1,000 dilution; Cell Signaling Technology, Inc.), E-cadherin (cat. no. 3195; 1:1,000 dilution; Cell Signaling Technology, Inc.), KLF-2 (cat. no. ab203591; 1:500 dilution; Abcam, Cambridge, MA, USA), and human $\beta$-actin (cat no. 3700; 1:1,000 dilution; Cell Signaling Technology, Inc.). Anti-rabbit IgG (cat. no. 7074; 1:3,000 dilution; Cell Signaling Technology, Inc.) and antimouse IgG (cat. no. 7076; 1:3,000 dilution; Cell Signaling Technology, Inc.) horseradish peroxidase-conjugated secondary antibodies were used for $2 \mathrm{~h}$ at room temperature. The enhanced chemiluminescence kit (Beyotime Institute of Biotechnology) was used to detect results of western blot analysis. The relative level of each protein was deduced from the ratio of the mean value of each band to that of $\beta$-actin. The relative densities were quantified with a digital imaging analyzer, ImageJ 1.4.1 (National Institutes of Health, Bethesda, MA, USA).

Statistical analysis. All of the statistical analyses were performed using SPSS 23.0 software (IBM Corp., Armonk, NY, USA). Disease-free survival rates were evaluated using Kaplan-Meier curves, and the Cox proportional hazards regression model was used to evaluate the hazard ratio (HR). Log-rank test is used to compare two or more groups by testing the null hypothesis.
The null hypothesis states that the populations do not differ in the probability of an event at any time-point. Thus, log-rank test is the most commonly-used statistical test to compare the survival functions of two or more groups. Kaplan Meier and Cox regression analyses were used to assess the association between LINC00673 and overall survival, and prognosis. Data are presented as the mean \pm standard deviation. The differences in characteristics between two groups were examined by the Student's t-test. The differences in characteristics between three groups were examined by analysis of variance, and the least significant difference test was applied to detect the differences between each pair of groups. The expression difference between two groups from TCGA database was used by Mann-Whitney $U$ test. Clinicopathological characteristics were evaluated using the $\chi^{2}$ test. Pearson correlation analysis was used to evaluate correlation between LINC00673 and KLF-2 expression in validate cohort. $\mathrm{P}<0.05$ was considered to indicate a statistically significant difference.

\section{Results}

Upregulation of LINC00673 in thyroid carcinoma tissues and the associated between LINC00673 expression and clinical features. To validate the RNA sequencing results (unpublished data), the expression levels of LINC00673 in 60 matched pairs of thyroid carcinoma and adjacent non-tumor tissues, 1 normal thyroid cell line and 3 thyroid carcinoma cell lines were examined using RT-qPCR. The results indicated that LINC00673 expression was higher in tumor tissues than in adjacent normal tissues $(\mathrm{P}<0.05$; Fig. 1A). To further understand the association between LINC00673 and thyroid carcinoma, the patients were divided into low-expression and high-expression groups 
Table I. Association between LINC00673 and clinicopathologic characteristics in the validated cohort.

\begin{tabular}{|c|c|c|c|c|}
\hline Clinicopathologic characteristics & Low expression $(\%)$ & High expression $(\%)$ & $\chi^{2}$ & P-value \\
\hline Age & & & 1.086 & 0.297 \\
\hline$\leq 45$ & $15(50)$ & $11(36.7)$ & & \\
\hline$>45$ & $15(50)$ & $19(63.3)$ & & \\
\hline Sex & & & 2.5 & 0.114 \\
\hline Female & $15(50)$ & $21(70)$ & & \\
\hline Male & $15(50)$ & $9(30)$ & & \\
\hline Tumor size & & & 5.934 & $0.015^{\mathrm{a}}$ \\
\hline$\leq 10 \mathrm{~mm}$ & $15(50)$ & $6(20)$ & & \\
\hline$>10 \mathrm{~mm}$ & $15(50)$ & $24(80)$ & & \\
\hline Unilateral or bilateral & & & 0.069 & 0.793 \\
\hline Unilateral & $18(60)$ & $17(56.7)$ & & \\
\hline Bilateral & $12(40)$ & $13(43.3)$ & & \\
\hline Extrathyroidal invasion & & & 1.118 & 0.29 \\
\hline Yes & $3(10)$ & $1(3.3)$ & & \\
\hline No & $27(90)$ & $29(96.7)$ & & \\
\hline Lymph node metastasis & & & 6.696 & $0.01^{\mathrm{a}}$ \\
\hline Yes & $11(36.7)$ & $21(70)$ & & \\
\hline No & $19(63.3)$ & $9(30)$ & & \\
\hline American Joint Cancer Committee stage & & & 0.287 & 0.592 \\
\hline $\mathrm{I}+\mathrm{II}$ & $20(66.7)$ & $18(60)$ & & \\
\hline $\mathrm{III}+\mathrm{IV}$ & $10(33.3)$ & $12(40)$ & & \\
\hline
\end{tabular}

${ }^{\mathrm{a}} \mathrm{P}<0.05 . \mathrm{n}=60$.

A

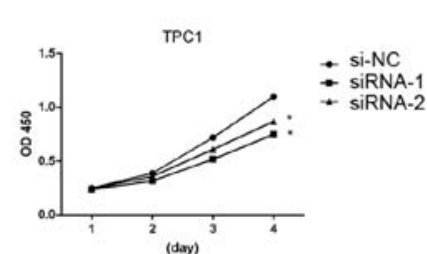

B

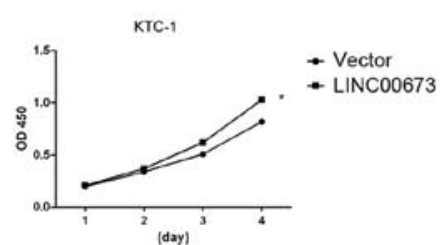

D

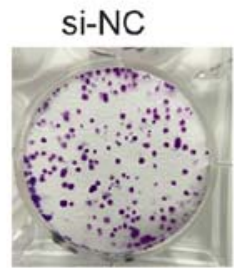

E

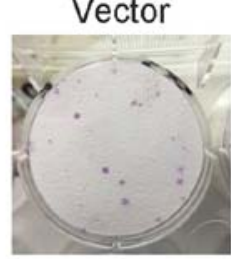

SiRNA-1

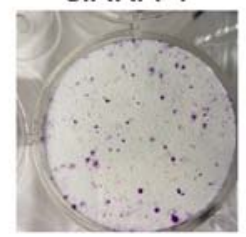

LINC00673

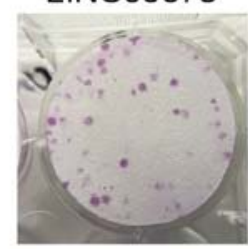

C

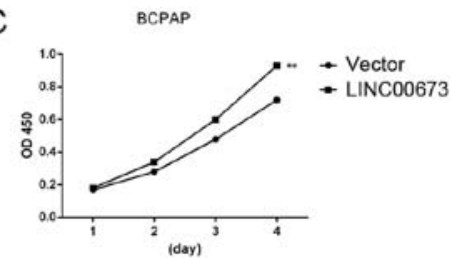

Figure 2. Dysregulation of LINC00673 gene expression in thyroid carcinoma cells inhibits proliferation and cell colony formation. TPC1 cells transfected with siRNA or si-NC were cultured in 96-well plates for 1-4 days, and then the CCK-8 assay was used to measure cell proliferation. Cell proliferation was significantly suppressed in thyroid carcinoma cells transfected with siRNA. (B) KTC-1 and (C) BCPAP cell lines transfected with LINC00673 or a vector control were cultured in 96-well plates for 1-4 days, and then the CCK-8 assay was used to measure cell proliferation. Cell proliferation was significantly enhanced in thyroid carcinoma cells transfected with LINC00673. (D) TPC1 cells transfected with siRNA or si-NC were cultured in 6-well plates for 10-14 days. (E) KTC-1 and (F) BCPAP cell lines transfected with LINC00673 or a vector control were cultured in 6 -well plates for $10-14$ days. ${ }^{*} \mathrm{P}<0.05$, $^{* *} \mathrm{P}<0.01$ vs. si-NC or a vector control using one-way analysis of variance. LINC00673, long intergenic non-protein coding RNA 673; si-NC, siRNA negative control; siRNA, small interfering RNA. 
A
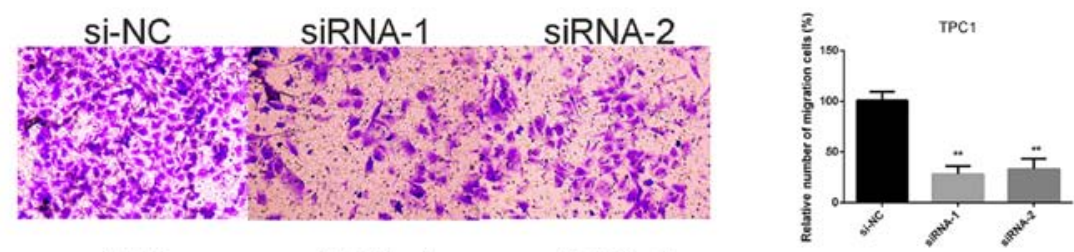

B
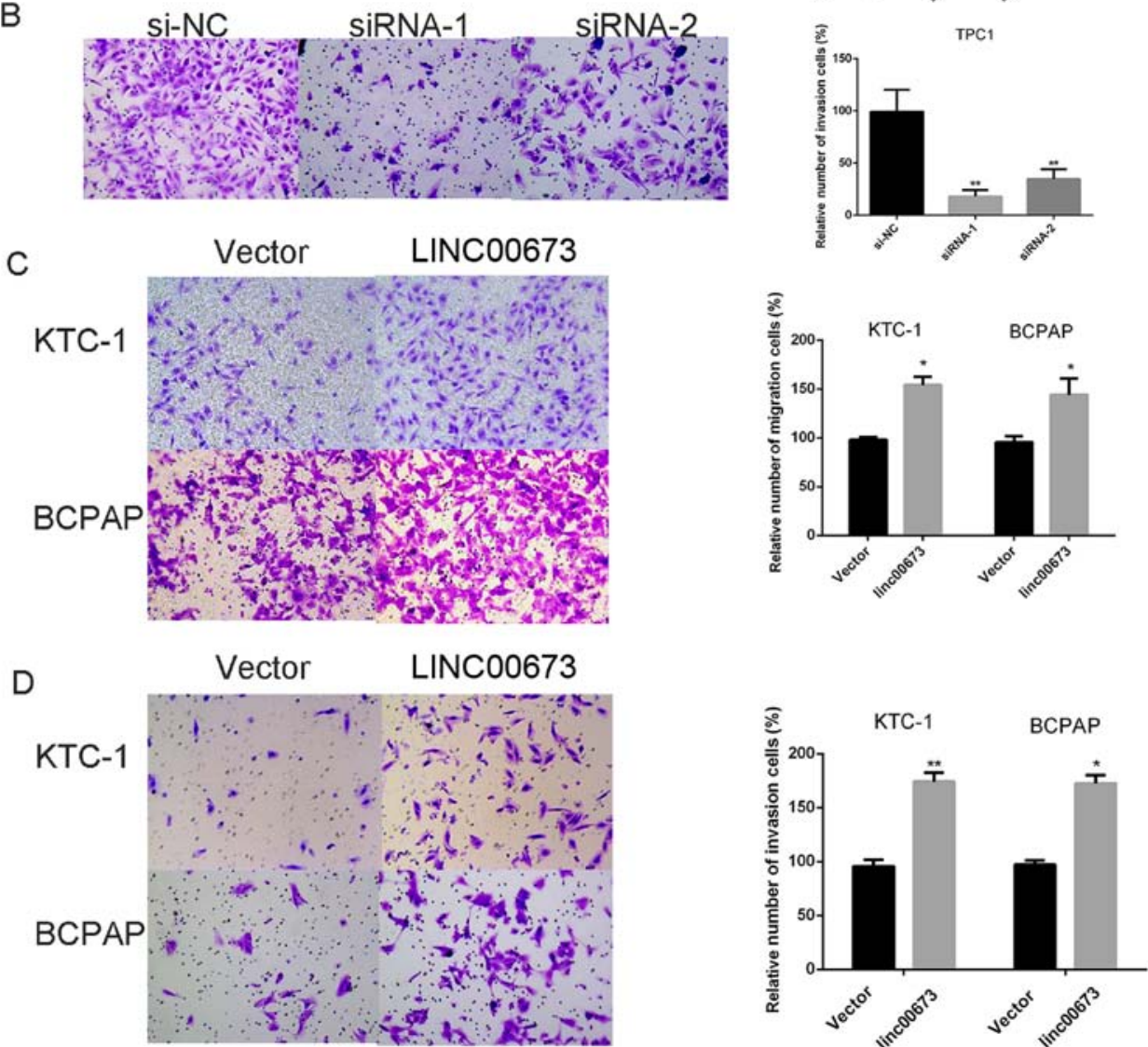

Figure 3. Dysregulation of LINC00673 gene expression in thyroid carcinoma cells inhibits migration and invasion. (A) In TPC1 cells, Transwell migration assays in LINC00673 knockdown cells and the corresponding control cells. (B) In TPC1 cells, Transwell invasion assays in LINC00673 knockdown cells and the corresponding control cells. (C) In KTC and BCPAP cells, Transwell migration assays in LINC00673 overexpression cells and the corresponding control cells. (D) In KTC and BCPAP cells, Transwell invasion assays in LINC00673 overexpression cells and the corresponding control cells. The columns represent the mean number of migrating or invading cells from at least three independent experiments. ${ }^{*} \mathrm{P}<0.05,{ }^{* *} \mathrm{P}<0.01$ vs. si-NC or vector using one-way analysis of variance. si-NC, siRNA negative control; siRNA, small interfering RNA; LINC00673, long intergenic non-protein coding RNA 673.

according to the median value in the validation cohort. As shown in Table I, tumor size $(\mathrm{P}=0.015)$ and lymph node metastasis $(\mathrm{P}=0.01)$ were significantly associated with LINC00673 expression. These results indicate that LINC00673 is upregulated in thyroid carcinoma and that it may function as an oncogene.

LINC00673 regulates the proliferation of thyroid carcinoma cell lines. As demonstrated in Fig. 1B, LINC00673 expression in the TPC1 cell line was significantly higher than that of the normal thyroid cell line HTORI-3, while the KTC-1 and BCPAP cells lines exhibited relatively low LINC00673 expression $(\mathrm{P}<0.05$; Fig. 1B). Subsequently, loss-of-function and gain-of-function experiments were performed to determine the role of LINC00673 in thyroid carcinoma. LINC00673 expression was knocked down in TPC1 cells using siRNA, and LINC00673 was overexpressed in KTC-1 and BCPAP cells using a pcDNA vector (Fig. 1C and D). The CCK-8 assay and colony formation experiments were performed to assess the effects of LINC00673 on thyroid carcinoma cell proliferation.
As demonstrated in Fig. 2A-C, thyroid carcinoma cell proliferation was significantly inhibited in TPC1 cells by knocking down LINC00673 expression (transfected with siRNA-1 or siRNA-2) compared with the control cells (transfected with si-NC); whereas, upregulation of LINC00673 enhanced KTC-1 and BCPAP cell proliferation compared with the vector control. Cell colony formation was also significantly inhibited in TPC1 cells by knocking down LINC00673 expression compared with control cells; whereas overexpression of LINC00673 exhibited the opposite effect in KTC-1 and BCPAP cells (Fig. 2D-F).

LINC00673 regulates the metastasis of thyroid carcinoma cell lines by modulating epithelial-mesenchymal transition (EMT). Given the association between LINC00673 expression and clinicopathological features, the role of LINC00673 in the migration and invasion of TPC cell lines was investigated. The results showed that downregulated LINC00673 weakened migration and invasion of TPC1 cells compared with control cells (Fig. 3A and B); whereas overexpression of LINC00673 


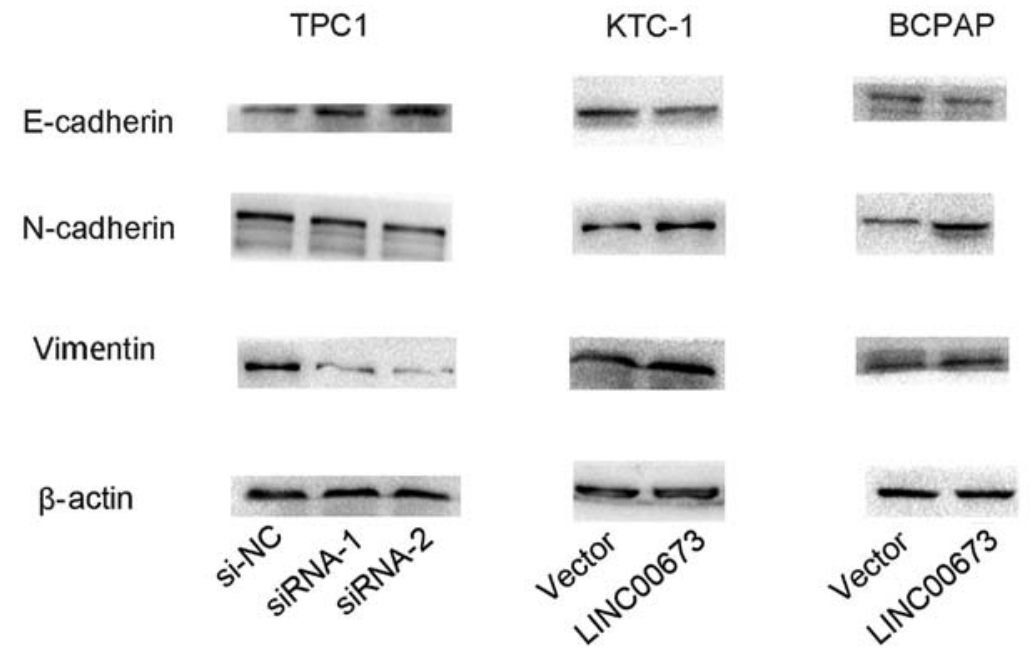

Figure 4. Effect of LINC00673 on epithelial-mesenchymal transition proteins in thyroid carcinoma cell lines (TPC1, KTC-1 and BCPAP). E-cadherin $\mathrm{N}$-cadherin, and vimentin protein expression determined by western blotting in TPC1 cells transfected with siRNA or si-NC, and KTC-1 and BCPAP cells transfected with empty vector or LINC00673 overexpression vector. si-NC, siRNA negative control; siRNA, small interfering RNA; LINC00673, long intergenic non-protein coding RNA 673.

A

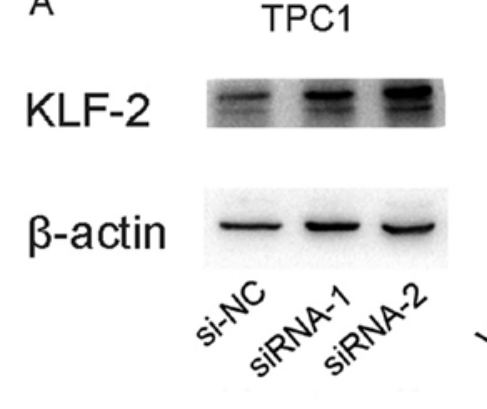

C

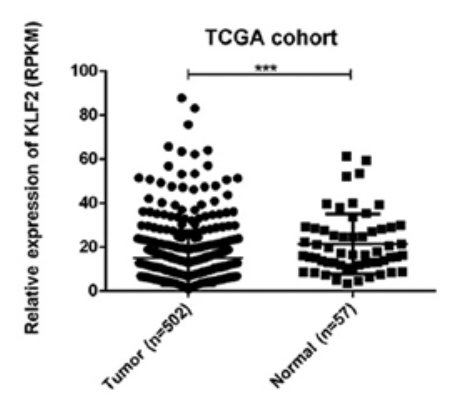

KTC-1

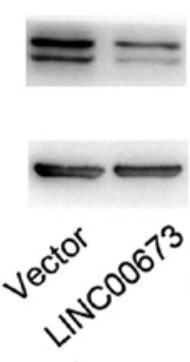

BCPAP

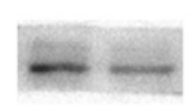

B

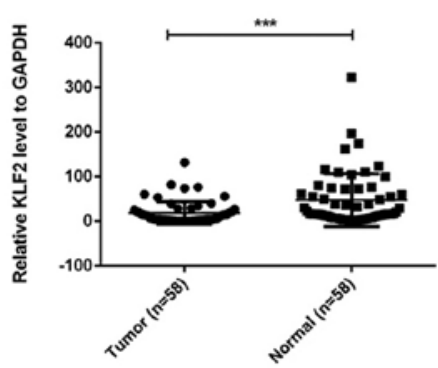

Figure 5. The expression of KLF-2 in a validated cohort of patients with thyroid carcinoma and in thyroid carcinoma cell lines. (A) Western blot analysis of the KLF-2 protein level after downregulation (siRNA) or upregulation of LINC00673. (B) KLF-2 expression was detected by RT-qPCR in 58 matched pairs of thyroid carcinoma and adjacent non-tumor tissues. (C) The Cancer Genome Atlas cohort contained 502 thyroid tumor tissues and 57 normal tissues. Reads per kilobase per million is used as the level of KLF-2. The data were analyzed using the Mann-Whitney U test. (D) Analysis of the relationship between LINC00673 expression ( $\Delta$ Cq value) and KLF-2 mRNA level ( $\Delta$ Cq value) in 45 thyroid carcinoma tissues. ${ }^{* * * *} \mathrm{P}<0.001$. RT-qPCR, reverse transcription-quantitative polymerase chain reaction; KLF-2, Kruppel-like factor 2; si-NC, small interfering RNA negative control; siRNA, small interfering RNA; LINC00673, long intergenic non-protein coding RNA 673.

had the opposite effect in KTC-1 and BCPAP cells (Fig. 3C and D). Previous studies have demonstrated that EMT is a key process during metastasis $(20,21)$. Thus, the protein levels of epithelial and mesenchymal markers were examined in thyroid carcinoma cell lines. As demonstrated in Fig. 4, knocking down LINC00673 reduced the expression of N-cadherin and vimentin, and enhanced E-cadherin expression; whereas, overexpression of LINC00673 had the opposite effect, suggesting that LINC00673 may regulate metastasis by altering EMT.
KLF-2 is a potential downstream target of LINC00673. Huang et al (22) reported that the oncogenic function of LINC00673 is partially dependent on repression of KLF2. In this study, knock down of LINC00673 enhanced the protein levels of KLF-2, while upregulation of LINC00673 inhibits the expression of KLF-2 (Fig. 5A). Additionally, KLF2 mRNA expression was assessed in 58 matched pairs of thyroid carcinoma tumor samples and adjacent normal tissues by RT-qPCR, which demonstrated that KLF-2 has lower expres- 
Table II. Association between KLF-2 and clinicopathologic characteristics in the validated cohort.

\begin{tabular}{|c|c|c|c|c|}
\hline Clinicopathologic characteristics & Low expression $(\%)$ & High expression $(\%)$ & $\chi^{2}$ & P-value \\
\hline Age & & & 1.086 & 0.297 \\
\hline$\leq 45$ & $11(36.7)$ & $15(50)$ & & \\
\hline$>45$ & $19(63.7)$ & $15(50)$ & & \\
\hline Sex & & & 0 & 1 \\
\hline Female & $18(60)$ & $18(60)$ & & \\
\hline Male & $12(40)$ & $12(40)$ & & \\
\hline Tumor size & & & 9.32 & $0.002^{\mathrm{a}}$ \\
\hline$\leq 10 \mathrm{~mm}$ & $4(13.3)$ & $15(50)$ & & \\
\hline$>10 \mathrm{~mm}$ & $26(86.7)$ & $15(50)$ & & \\
\hline Unilateral or bilateral & & & 1.714 & 0.19 \\
\hline Unilateral & $15(50)$ & $20(66.7)$ & & \\
\hline Bilateral & $15(50)$ & $10(33.3)$ & & \\
\hline Extrathyroidal invasion & & & 0 & 1 \\
\hline Yes & $2(6.7)$ & $2(6.7)$ & & \\
\hline No & $28(93.3)$ & $28(93.3)$ & & \\
\hline Lymph node metastasis & & & 2.411 & 0.121 \\
\hline Yes & $19(63.3)$ & $13(43.3)$ & & \\
\hline No & $11(36.7)$ & $17(56.7)$ & & \\
\hline American Joint Cancer Committee stage & & & 0.287 & 0.592 \\
\hline $\mathrm{I}+\mathrm{II}$ & $18(60)$ & $20(66.7)$ & & \\
\hline III+IV & $12(40)$ & $10(33.3)$ & & \\
\hline
\end{tabular}

${ }^{\mathrm{a}} \mathrm{P}<0.05 . \mathrm{n}=60$.

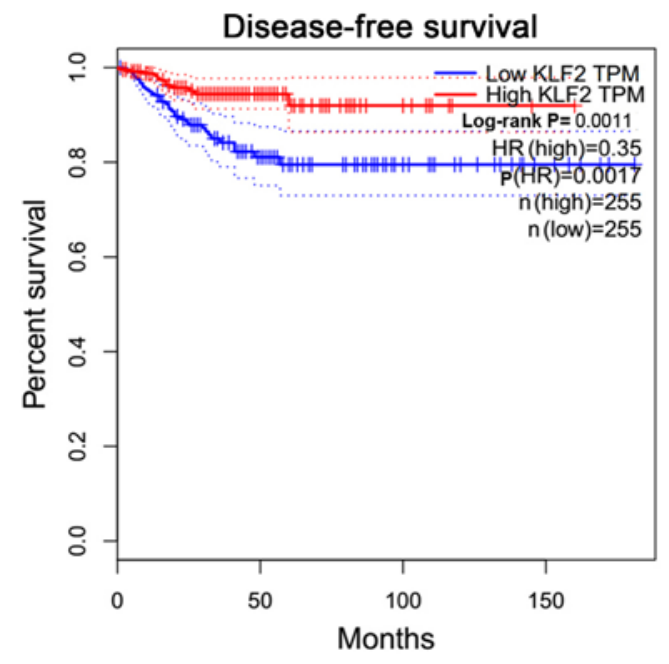

Figure 6. Kaplan-Meier analysis of disease-free survival of patients with thyroid carcinoma in the Cancer Genome Atlas cohort with different levels of KLF-2 expression. Patients with lower KLF-2 expression had a shorter disease-free survival time compared with patients with higher KLF-2 expression. KLF-2, Kruppel-like factor 2; HR, hazard ratio; TPM, transcripts per million.

sion in thyroid carcinoma tumor samples than that in normal samples (Fig. 5B). The same trend was also observed in a TCGA cohort (Fig. 5C). Pearson correlation analysis indicated a negative correlation between LINC00673 and KLF-2 expres- sion in 50 paired clinical thyroid carcinoma tissues (Fig. 5D). These results suggest that KLF-2 is a potential downstream target of LINC00673.

Association between KLF-2 expression and patient clinicopathologic features. To determine whether KLF-2 expression is involved in thyroid carcinoma tumorigenesis, the association between KLF-2 and patient clinical features was evaluated. The validated cohort was divided into a high-expression group and a low-expression group according to KLF-2 median expression level. As presented in Table II, patients with a lower expression level of KLF-2 had a larger tumor size than in the high expression group $(\mathrm{P}=0.002)$. In the TCGA cohort (Table III), the results revealed that low KLF-2 expression was associated with tumor size $(\mathrm{P}<0.001)$, lymph node metastasis $(\mathrm{P}=0.007)$, histological type $(\mathrm{P}=0.001)$ and $\mathrm{T}$ stage $(\mathrm{P}<0.001)$. As presented in Fig. 6, the difference between the survival curves of the two groups was statistically significant $(\mathrm{P}=0.0011)$. This finding indicated that low KLF-2 expression was associated with an unfavorable prognosis in patients with thyroid carcinoma.

Restoration of KLF-2 induces thyroid carcinoma cell proliferation and metastasis. As shown in Fig. 7A, KLF-2 expression in the TPC1, KTC-1 and BCPAP cell lines was significantly lower than that of the HTORI-3 cell line. To further confirm whether KLF-2 influences the progression of thyroid carcinoma, KLF-2 

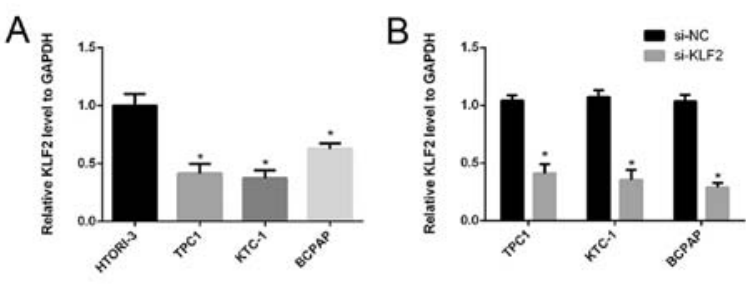

D

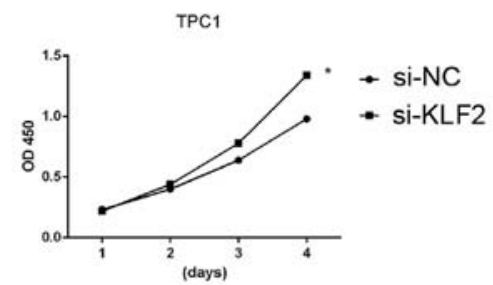

$E$
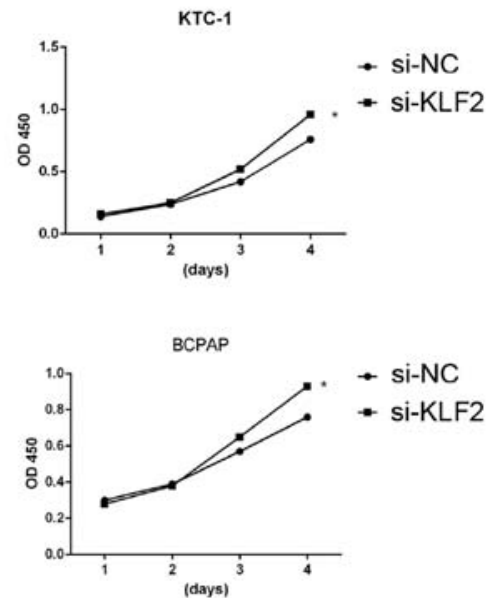

TPC1

C TPC1 KTC-1 BCPAP
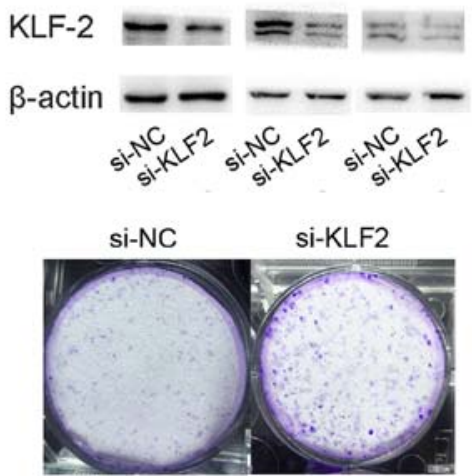

KTC-1

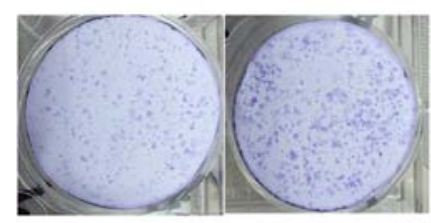

BCPAP

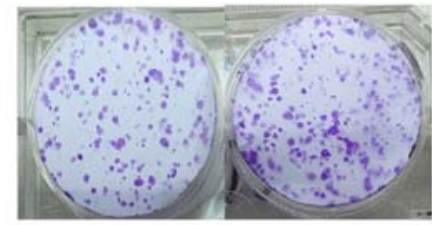

Figure 7. Downregulation of KLF-2 enhances thyroid carcinoma cell line proliferation and cell colony formation. (A) The relative expression of KLF-2 (compared with the GAPDH gene) was examined via RT-qPCR in a normal thyroid cell line (HTORI-3), and thyroid carcinoma cell lines (TPC1, KTC-1 and BCPAP). (B) RT-qPCR analysis and (C) western blot analysis of KLF-2 mRNA and protein levels in si-NC and si-KLF2 transfected cells. (D) Cell proliferation assay: TPC1, KTC-1 and BCPAP cell lines transfected with si-KLF2 or si-NC were cultured in 96-well plates for 1-4 days, and then cell proliferation was measured using the CCK-8 assay. Cell proliferation was significantly enhanced in TPC1, KTC-1 and BCPAP cells transfected with si-KLF2. (E) TPC1, KTC-1 and BCPAP cell lines transfected with si-KLF2 or si-NC were cultured in 6-well plates for 10-14 days. "P<0.05 vs. si-NC; comparison to cells treated with si-NC using Student's t-test. KLF-2, Kruppel-like factor 2; si, small interfering RNA; NC, negative control; OD, optical density.

A

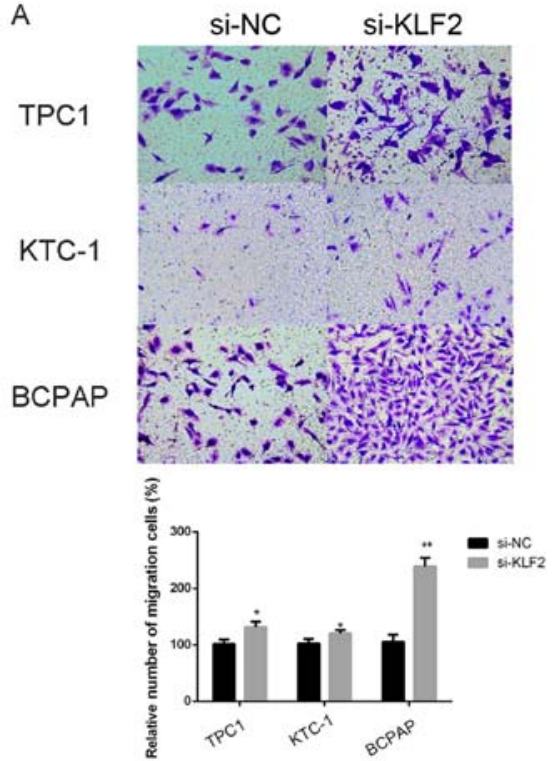

B

TPC1

KTC-1
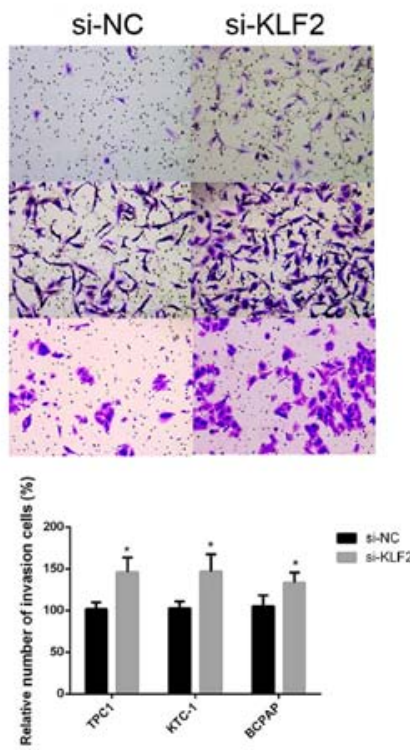

Figure 8. Downregulation of KLF-2 enhances the migration and invasion of thyroid carcinoma cell lines. (A) The number of migrating cells was higher in TPC1, KTC-1 and BCPAP cell lines transfected with si-KLF2 than in cells transfected with si-NC. (B) The number of invading cells was higher in TPC1, KTC-1 and BCPAP cell lines transfected with si-KLF2 than in cells transfected with si-NC. ${ }^{*} \mathrm{P}<0.05,{ }^{* * *} \mathrm{P}<0.01$ vs. si-NC using Student's t-test. si, small interfering RNA; NC, negative control; KLF-2, Kruppel-like factor 2. 
Table III. Association between KLF-2 and clinicopathologic characteristics in The Cancer Genome Atlas cohort.

\begin{tabular}{|c|c|c|c|c|}
\hline Clinicopathologic characteristics & Low expression $(\%)$ & High expression $(\%)$ & $\chi^{2}$ & P-value \\
\hline Age & & & 0 & 1 \\
\hline$\leq 45$ & 115 & 115 & & \\
\hline$>45$ & 130 & 130 & & \\
\hline $\operatorname{Sex}$ & & & 0.263 & 0.608 \\
\hline Female & 178 & 183 & & \\
\hline Male & 67 & 62 & & \\
\hline Histological type & & & 16.803 & $0.001^{\mathrm{a}}$ \\
\hline Classical & 184 & 164 & & \\
\hline Follicular & 33 & 66 & & \\
\hline Tall cell & 23 & 11 & & \\
\hline Other & 5 & 4 & & \\
\hline Tumor size & & & 13.871 & $0.000^{\mathrm{a}}$ \\
\hline$\leq 20 \mathrm{~mm}$ & 50 & 87 & & \\
\hline$>20 \mathrm{~mm}$ & 195 & 158 & & \\
\hline T stage & & & 13.871 & $0.000^{\mathrm{a}}$ \\
\hline $\mathrm{T} 1$ & 50 & 87 & & \\
\hline$>\mathrm{T} 1$ & 195 & 158 & & \\
\hline Extrathyroidal invasion & & & 0.207 & 0.649 \\
\hline Yes & 111 & 106 & & \\
\hline No & 134 & 139 & & \\
\hline Lymph node metastasis & & & 7.401 & $0.007^{\mathrm{a}}$ \\
\hline Yes & 148 & 118 & & \\
\hline No & 97 & 127 & & \\
\hline American Joint Cancer Committee stage & & & 2.641 & 0.104 \\
\hline $\mathrm{I}+\mathrm{II}$ & 154 & 171 & & \\
\hline III+IV & 91 & 74 & & \\
\hline
\end{tabular}

${ }^{\text {ap }}<0.05$. Twelve samples were excluded due to lack of sufficient clinical data. $\mathrm{n}=490$.

expression was knocked down in the TPC1, KTC-1 and BCPAP cell lines using siRNA and confirmed the knockdown efficiency at the RNA and protein levels (Fig. 7B and C). The results from the validated and TCGA cohorts suggest that the expression of KLF-2 is associated with tumor size; thus, we hypothesized that abnormal expression of KLF-2 may influence the proliferation of thyroid carcinoma cells. The CCK-8 and colony formation assays were performed, and the results demonstrated that knockdown of KLF-2 enhanced the proliferation of TPC1, KTC-1 and BCPAP cell lines (Fig. 7D and E). Transwell migration and invasion assays were then performed to examine the metastatic ability of the thyroid carcinoma cell lines. As presented in Fig. 8A, the number of migrating cells was significantly increased by knocking down KLF-2 expression in the cell lines. Consistent with previous findings, the invasion ability was induced in thyroid carcinoma cell lines by knocking down KLF-2 expression compared with the control group (Fig. 8B).

Oncogenic role of LINC00673 is partly dependent on repressing $K L F-2$ expression. To determine whether KLF-2 influences the LINC00673-associated enhancement of proliferation and metastasis in thyroid carcinoma cells, rescue experiments were performed. TPC1 and BCPAP cells were co-transfected with LINC00673 and KLF2 siRNAs. CCK-8 assay and Transwell assays demonstrated that knocking down KLF2 partially rescued the LINC00673 silencing-mediated effects on cell growth and invasion/metastasis in TPC1 and BCPAP cells (Fig. 9). As demonstrated in Fig. 10, co-transfection with LINC00673 and KLF2 siRNA partially rescued the effects of LINC00673 downregulation on KLF-2 and E-cadherin upregulation, and the downregulation mesenchymal markers, N-cadherin and vimentin, was also partially rescued. These results suggest that KLF-2 is an important mediator of LINC00673-associated cell proliferation, metastasis and EMT.

\section{Discussion}

Thyroid cancer is one of the fastest growing malignancies worldwide with a rate of $4 \%$ per year (23). Despite extensive research on IncRNAs and thyroid carcinoma (24-26), the underlying molecular mechanisms responsible for the function of lncRNAs in thyroid carcinoma are not well understood.

In a previously unpublished study, we selected 19 matched pairs of thyroid carcinomatumortissues and adjacent non-cancer 
A
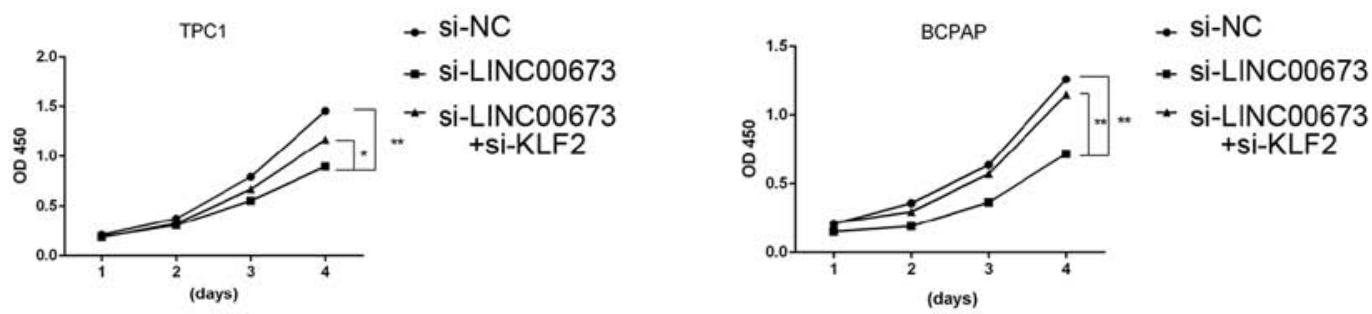

B
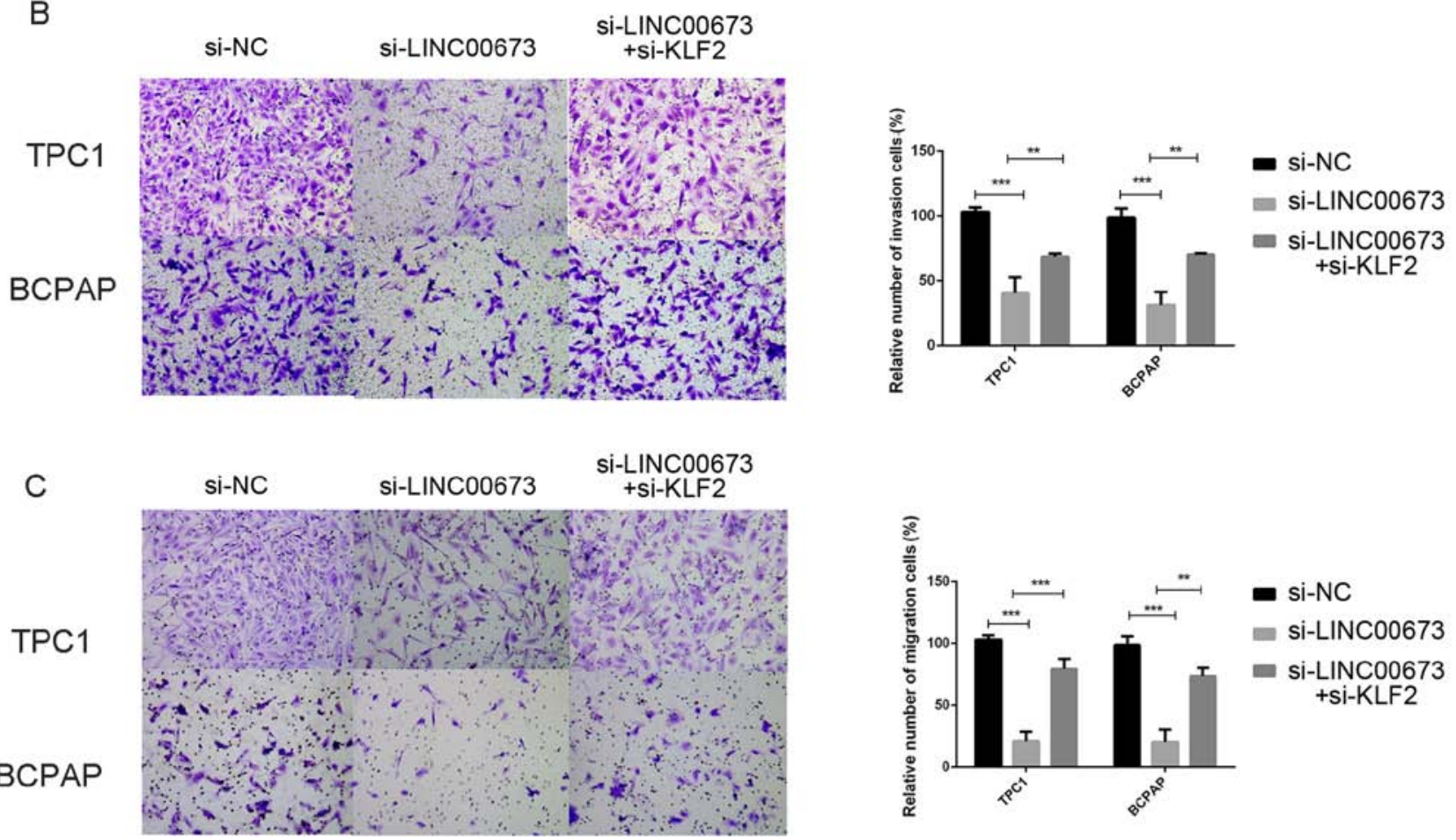

Figure 9. The oncogenic role of LINC00673 is partially dependent on repressing KLF-2 expression. (A) CCK-8 assays were performed to determine the viability of TPC1 and BCPAP cells transfected with si-NC, si-LINC00673 or si-LINC00673+si-KLF2. (B) Transwell migration assays were performed to determine the viability of TPC1 and BCPAP cells transfected with si-NC, si-LINC00673 or si-LINC00673+si-KLF2. (C) Transwell invasion assays were performed to determine the viability of TPC1 and BCPAP cells transfected with si-NC, si-LINC00673 or si-LINC00673+si-KLF2. ${ }^{*} \mathrm{P}<0.05,{ }^{* *} \mathrm{P}<0.01,{ }^{* * * *} \mathrm{P}<0.001$. si, small interfering RNA; NC, negative control; LINC00673, long intergenic non-protein coding RNA 673; KLF-2, Kruppel-like factor 2.

TPC1

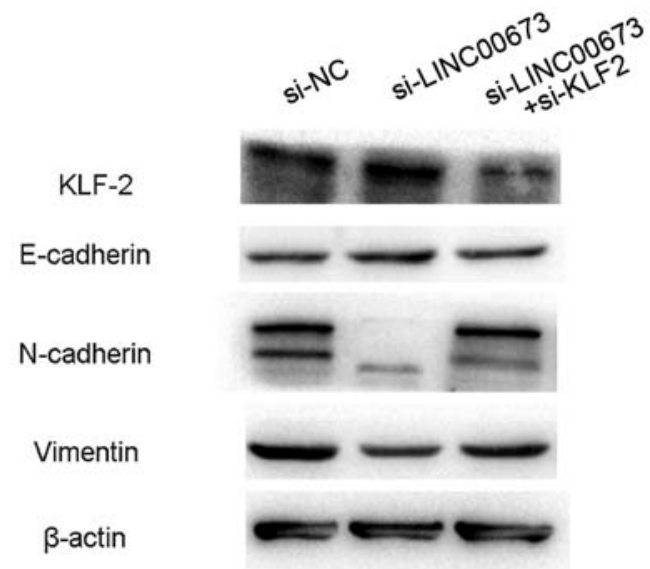

\section{BCPAP}

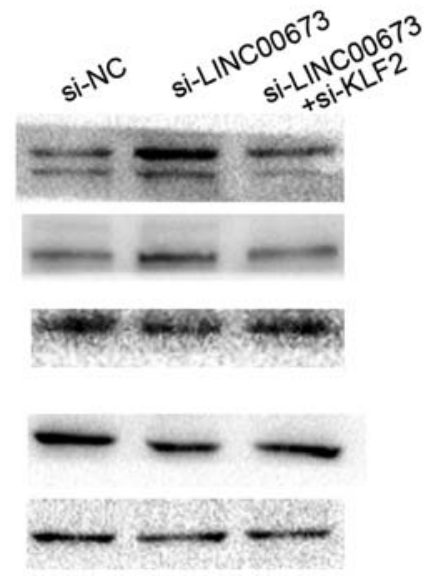

Figure 10. Ability of LINC00673 to influence epithelial-mesenchymal transition is partially dependent on the repression of KLF-2 expression. Western blot analysis was performed to determine the protein levels of KLF-2, E-cadherin, N-cadherin and vimentin in cells transfected with si-NC, si-LINC00673 or si-LINC00673+si-KLF2. si, small interfering RNA; NC, negative control; LINC00673, long intergenic non-protein coding RNA 673; KLF-2, Kruppel-like factor 2 . 
tissues to conduct whole transcriptome resequencing, and the results revealed that LINC00673 expression was higher in tumor tissues than in adjacent normal thyroid tissues. Ma et al (27) reported that LINC00673 binds to histone-lysine $\mathrm{N}$-methyltransferase EZH2 and silences homeobox protein HOXA5 to promote non-small cell lung cancer metastasis. However, the association between LINC00673 and tumorigenesis remains unclear. The findings in this study demonstrated that LINC00673 expression is higher in thyroid tumor tissues and that patients with higher LINC00673 have larger tumors and increased lymph node metastasis compared with normal tissues. Loss-of-function and gain-of-function experiments were performed to demonstrate the function of LINC00673 in cell proliferation and metastasis. The findings also indicated that knocking down LINC00673 may enhance the protein level of E-cadherin, and inhibit expression of $\mathrm{N}$-cadherin and vimentin, while upregulation of LINC00673 had the opposite effect. In addition, knock down of LINC00673 enhanced the protein levels of KLF-2, and upregulation of LINC00673 inhibited KLF-2 expression.

KLF-2 is located at chromosome 19p13.11 and is $~ 2,800 \mathrm{bp}$ in length. Although previous studies have reported that KLF-2 is dysregulated in cancer and has an influence on tumorigenesis in various cancer tissues (28-30), very little is known about its biological role and the mechanism of action of KLF-2 in thyroid carcinoma. In the current study, it was demonstrated that the expression of KLF-2 is lower in thyroid tumor tissues than that in normal tissues, in a validated cohort and in a TCGA cohort. The expression of KLF-2 was negatively correlated with the expression of LINC00673 in the validated cohort, and lower KLF-2 expression was associated with increased lymph node metastasis and a larger tumor size. KLF-2 downregulation markedly enhanced the proliferation and metastasis of thyroid cancer cells. Knocking down KLF2 partially rescued the effects of LINC00673 silencing and resulted in increased thyroid carcinoma cell growth and metastasis. These results suggest that LINC00673 functions as an oncogene in thyroid carcinoma, partially by inhibiting KLF-2 expression.

Despite these findings, some limitations do exist. The number of patients included in the validated cohort should be increased to provide more convincing results. Additionally, in vivo experiments should be conducted to validate the oncogenic effects of LINC00673.

In summary, LINC00673 is upregulated in thyroid carcinoma, and the abnormal expression may exert an oncogenic functional role in thyroid carcinoma. The findings of this study provide a potential new marker and target for gene therapy in thyroid carcinoma.

\section{Acknowledgements}

The authors would like to thank all the doctors of Department of Thyroid and Breast Surgery, The First Affiliated Hospital of Wenzhou Medical University (Wenzhou, China), for providing all the necessary information required for this study.

\section{Funding}

This study was funded by the National Natural Science Foundation of China (no. 81572291), the Natural Science
Foundation of Zhejiang province (nos. LY17H160053, LGF18H160031, GF18H160071 and LGF18H160032), the Medical and Health Technology Projects of Zhejiang province (no. 2017187475) and the Science and Technology Project of Wenzhou (no. Y20170030).

\section{Availability of data and materials}

The data sets supporting the conclusions of the present study are included in this published article. The raw data stored on the main electronic data storage system of the First Affiliated Hospital of Wenzhou Medical University are available from the corresponding author on reasonable request.

\section{Authors' contributions}

$\mathrm{AB}$ analyzed the raw data and wrote the original draft. $\mathrm{AB}$ and EJX investigated and interpreted the data. YYS and XFZ collected the raw data. OCW conceptualized and designed the study and provided supervision. All authors read and approved the final manuscript.

\section{Ethics approval and consent to participate}

Ethical approval for this study was obtained from the Ethics Committee of the First Affiliated Hospital of Wenzhou Medical University. Written informed consent was obtained from each individual participant.

\section{Patient consent for publication}

Not applicable.

\section{Competing interests}

The authors declare that they have no competing interests.

\section{References}

1. Siegel RL, Miller KD and Jemal A: Cancer statistics, 2018. CA Cancer J Clin 68: 7-30, 2018.

2. Burns WR and Zeiger MA: Differentiated thyroid cancer. Semin Oncol 37: 557-566, 2010.

3. Fagin JA and Wells SA Jr: Biologic and clinical perspectives on thyroid cancer. N Engl J Med 375: 1054-1067, 2016.

4. Prescott JD and Zeiger MA: The RET oncogene in papillary thyroid carcinoma. Cancer 121: 2137-2146, 2015.

5. Jin Y, Jin W, Zheng Z, Chen E, Wang Q, Wang Y, Wang O and Zhang X: GABRB2 plays an important role in the lymph node metastasis of papillary thyroid cancer. Biochem Biophys Res Commun 492: 323-330, 2017.

6. Gianoukakis AG, Flores NM, Pelletier CL, Forsythe A, Wolfe GR and Taylor MH: Treatment patterns, health state, and health care resource utilization of patients with radioactive iodine refractory differentiated thyroid cancer. Cancer Manag Res 8: 67-76, 2016.

7. Yue B, Cai D, Liu C, Fang C and Yan D: Linc00152 functions as a competing endogenous RNA to confer oxaliplatin resistance and holds prognostic values in colon cancer. Mol Ther 24: 2064-2077, 2016.

8. Qian K, Liu G, Tang Z, Hu Y, Fang Y, Chen Z and Xu X: The long non-coding RNA NEAT1 interacted with miR-101 modulates breast cancer growth by targeting EZH2. Arch Biochem Biophys 615: 1-9, 2017.

9. Peng J, Zhang L, Yuan C, Zhou L, Xu S, Lin Y, Zhang J, Yin W and $\mathrm{Lu} \mathrm{J}$ : Expression profile analysis of long noncoding RNA in ER-positive subtype breast cancer using microarray technique and bioinformatics. Cancer Manag Res 9: 891-901, 2017. 
10. Gong Z, Zhang S, Zhang W, Huang H, Li Q, Deng H, Ma J, Zhou M, Xiang J, Wu M, et al: Long non-coding RNAs in cancer. Sci China Life Sci 55: 1120-1124, 2012.

11. Gou X, Zhao X and Wang Z: Long noncoding RNA PVT1 promotes hepatocellular carcinoma progression through regulating miR-214. Cancer Biomark 20: 511-519, 2017.

12. Wen X, Han XR, Wang YJ, Fan SH, Zhuang J, Zhang ZF, Shan Q, Li MQ, Hu B, Sun CH, et al: Effects of long noncoding RNA SPRY4-IT1-mediated EZH2 on the invasion and migration of lung adenocarcinoma. J Cell Biochem 119: 1827-1840, 2018.

13. Dai W, Tian Y, Jiang B and Chen W: Down-regulation of long non-coding RNA AFAP1-AS1 inhibits tumor growth, promotes apoptosis and decreases metastasis in thyroid cancer. Biomed Pharmacother 99: 191-197, 2018.

14. Qin Y, Sun W, Zhang H, Zhang P, Wang Z, Dong W, He L, Zhang T, Shao L, Zhang W, et al: lncRNA GAS8-AS1 inhibits cell proliferation through ATG5-mediated autophagy in papillary thyroid cancer. Endocrine 59: 555-564, 2018.

15. Ding S, Qu W, Jiao Y, Zhang J, Zhang C and Dang S: lncRNA SNHG12 promotes the proliferation and metastasis of papillary thyroid carcinoma cells through regulating wnt/beta-catenin signaling pathway. Cancer Biomark.22: 217-226, 2018.

16. Lu W, Zhang H, Niu Y, Wu Y, Sun W, Li H, Kong J, Ding K, Shen HM, Wu H, et al: Long non-coding RNA linc00673 regulated non-small cell lung cancer proliferation, migration, invasion and epithelial mesenchymal transition by sponging miR-150-5p. Mol Cancer 16: 118, 2017.

17. Abdul-Rahman U, Győrffy B and Adams BD: linc00673 (ERRLR01) is a prognostic indicator of overall survival in breast cancer. Transcription 9: 17-29, 2018.

18. Yu J, Liu Y, Gong Z, Zhang S, Guo C, Li X, Tang Y, Yang L, He Y1, Wei F, et al: Overexpression long non-coding RNA LINC00673 is associated with poor prognosis and promotes invasion and metastasis in tongue squamous cell carcinoma. Oncotarget 8: 16621-16632., 2017.

19. Livak KJ and Schmittgen TD: Analysis of relative gene expression data using real-time quantitative PCR and the 2(-Delta Delta C(T)) Method. Methods 25: 402-408, 2001

20. Thiery JP, Acloque H, Huang RY and Nieto MA: Epithelialmesenchymal transitions in development and disease. Cell 139: 871-890, 2009.

21. Li Q, Zhang C, Chen R, Xiong H, Qiu F, Liu S, Zhang M, Wang F, Wang Y, Zhou X, et al: Disrupting MALAT1/miR-200c sponge decreases invasion and migration in endometrioid endometrial carcinoma. Cancer Lett 383: 28-40, 2016.
22. Huang M, Hou J, Wang Y, Xie M, Wei C, Nie F, Wang Z and Sun M: Long noncoding RNA LINC00673 is Activated by SP1 and exerts oncogenic properties by interacting with LSD1 and EZH2 in gastric cancer. Mol Ther 25: 1014-1026, 2017.

23. Rahib L, Smith BD, Aizenberg R, Rosenzweig AB, Fleshman JM and Matrisian LM: Projecting cancer incidence and deaths to 2030: The unexpected burden of thyroid, liver, and pancreas cancers in the United States. Cancer Res 74: 2913-2921, 2014

24. Chu YH, Hardin H, Schneider DF, Chen H and Lloyd RV: MicroRNA-21 and long non-coding RNA MALAT1 are overexpressed markers in medullary thyroid carcinoma. Exp Mol Pathol 103: 229-236, 2017.

25. Lan X, Sun W, Dong W, Wang Z, Zhang T, He L and Zhang H: Downregulation of long noncoding RNA H19 contributes to the proliferation and migration of papillary thyroid carcinoma. Gene 646: 98-105, 2018.

26. Xia E, Bhandari A, Shen Y, Zhou X, Sindan N, Xiang J, Guan Y, Yang F and Wang O: lncRNA CCND2-AS1 promotes proliferation, migration, and invasion in papillary thyroid carcinoma. Biochem Biophys Res Commun 496: 628-632, 2018.

27. Ma C, Wu G, Zhu Q, Liu H, Yao Y, Yuan D, Liu Y, Lv T and Song Y: Long intergenic noncoding RNA 00673 promotes non-small-cell lung cancer metastasis by binding with EZH2 and causing epigenetic silencing of HOXA5. Oncotarget 8: 32696-32705, 2017.

28. Xu TP, Liu XX, Xia R, Yin L, Kong R, Chen WM, Huang MD and Shu YQ: SP1-induced upregulation of the long noncoding RNA TINCR regulates cell proliferation and apoptosis by affecting KLF2 mRNA stability in gastric cancer. Oncogene 34: 5648-5661, 2015 .

29. Xiong Y, Lingrel JB, Wüthrich M, Klein BS, Vasudevan NT, Jain MK, George M and Deepe GS Jr: Transcription factor KLF2 in dendritic cells downregulates Th2 programming via the HIF-1 $\alpha /$ Jagged2/Notch Axis. MBio 7: 7, 2016.

30. Fang J, Sun CC and Gong C: Long noncoding RNA XIST acts as an oncogene in non-small cell lung cancer by epigenetically repressing KLF2 expression. Biochem Biophys Res Commun 478: 811-817, 2016

This work is licensed under a Creative Commons Attribution-NonCommercial-NoDerivatives 4.0 International (CC BY-NC-ND 4.0) License. 